\title{
Guided duration of anticoagulation after unprovoked venous thromboembolism using D-dimer testing
}

\author{
Francis Couturaud
}

Affiliation: GETBO (Groupe d'Etude de la Thrombose de Bretagne Occidentale), EA 3878, CIC INSERM 1412, IFR 148, GIRC (Groupe d'Investigation et de Recherche Clinique) Thrombose, Dept of Internal Medicine and Chest Diseases, University Hospital Centre La Cavale Blanche, European Brittany University, Brest, France.

Correspondence: Francis Couturaud, Dept of Internal Medicine and Chest Diseases, University Hospital Centre La Cavale Blanche, European Brittany University, 29609 Brest, Cedex, France.

E-mail: francis.couturaudachu-brest.fr

$\boldsymbol{0}$ @ERSpublications

D-dimer testing after venous thromboembolism is helpful to identify patients eligible for indefinite anticoagulation http://ow.ly/ZluRz

Venous thromboembolism (VTE) is a frequent, multifactorial and potentially life-threatening disease [1-3]. When VTE occurs in the absence of any major risk factor (i.e. surgery, prolonged immobilisation or trauma of the lower limb), the risk of recurrent VTE is high (i.e. about $10 \%$ at 1 year and $30 \%$ at 5 years) after anticoagulation therapy is stopped, and current guidelines recommended indefinite anticoagulation once a first episode of unprovoked VTE has occurred [1-8]. However, only one-third of these patients will benefit from indefinite anticoagulation whereas, in the others, the risk of anticoagulant therapy-related bleeding probably outweighs any risk of recurrent VTE after stopping anticoagulation therapy. Therefore, the determination of the individual risk of recurrent VTE among patients with unprovoked VTE remains a major issue. In this setting, PALARETI et al. [9] report, in this issue of the European Respiratory Journal, the results of a subanalysis of the "DULCIS" study, a large prospective cohort of 1010 patients with unprovoked VTE [10]; the main aim was to evaluate the accuracy of serial D-dimer assessment to predict the risk of recurrent VTE according to whether patients had unprovoked pulmonary embolism (PE) or deep vein thrombosis (DVT) [9].

The first important result of this study is that the annual incidence rate of recurrent VTE was markedly higher $(8-11 \%$ per year) in patients with positive D-dimer test who did not resume anticoagulation as compared to those with negative D-dimer who permanently stopped anticoagulation (3-4\% per year), whether the initial episode was isolated PE or DVT (with or without PE). However, the annual incidence rate of recurrent VTE in patients with negative D-dimer was not low, particularly if VTE was isolated PE or occurred in the absence of a weak risk factor, such as minor trauma, leg injury or reduced mobility, or after 70 years. Similar observations of a non-high risk of recurrent VTE in patients with negative D-dimer test have been previously reported $[11,12]$. In the first major randomised trial having evaluated the accuracy of D-dimer to predict the risk of recurrent VTE in patients with a first episode of unprovoked VTE, patients with elevated D-dimer level 1 month after stopping anticoagulation were found to have a high risk of recurrence (incidence rate of $10.9 \%$ per year) in the group allocated to not resume anticoagulation whereas the incidence rate was low (2\% per year) in the group assigned to resume anticoagulation [11]; however, in patients with negative D-dimer test (for whom anticoagulation was permanently stopped), the risk of recurrence was moderate (i.e. $4.4 \%$ per year). In a prospective cohort of 319 patients presenting a first episode of unprovoked VTE, an overall incidence rate of recurrent VTE of $6.9 \%$ per year was observed in patients with two negative D-dimer results, except in young women on

Received: Jan 282016 | Accepted: March 082016

Conflict of interest: Disclosures can be found alongside the online version of this article at erj.ersjournals.com

Copyright @ERS 2016 
oestrogen-containing therapy, in whom no recurrent VTE was reported [12]. Therefore, this subanalysis of the DULCIS study provides additional evidence that the use of $\mathrm{D}$-dimer alone has a low discriminant power to identify patients at low risk of recurrent VTE. However, using D-dimer in combination with other clinical variables, such as age, sex or body mass index, appears accurate, although prospective validation is needed [13]. Conversely, positive D-dimer test results in patients with unprovoked VTE provide additional justification for indefinite anticoagulation therapy in patients with unprovoked VTE.

The second important finding is the observation of a higher risk of recurrence under the clinical presentation of $\mathrm{PE}$ in patients with an initial isolated PE as compared to those with DVT. Consistently, a recent meta-analysis [14], as well as randomised trials on the optimal duration of anticoagulation [5-8], reported similar results. As a consequence, for a similar risk of recurrent VTE after DVT or PE, the case-fatality rate of recurrent VTE after PE will be approximately four-fold higher than that after DVT, so that the benefit risk ratio of extended duration of anticoagulation is likely to be higher after PE than after DVT. This key point of the determination of the benefit/risk ratio of extended anticoagulation is unlikely to be significantly modified with novel oral anticoagulant (NOAC) therapy. Indeed, in the only study having directly compared warfarin with a NOAC, there was no significant reduction while on NOAC in terms of recurrent VTE, major bleeding or death, the only benefit being a reduction of the risk of clinically relevant non-major bleeding [15].

Finally, the present subanalysis conducted by PALARETI et al. [9] underlines some additional useful information: first, in patients with unprovoked VTE, the presence of negative D-dimer does not exclude a high risk of recurrent VTE, particularly in patients with isolated PE or those older than 70 years; secondly, a positive D-dimer assessment is strongly correlated with a high risk of recurrent VTE, which supports indefinite anticoagulation in most cases; thirdly, patients who had isolated PE are more likely to develop recurrence under the clinical presentation of PE as compared to patients with an initial DVT, and this observation also reinforces the need for indefinite anticoagulation in patients with unprovoked PE rather than in patients with unprovoked DVT.

\section{References}

Rosendaal FR. Venous thrombosis: a multicausal disease. Lancet 1999; 353: 1167-1173.

Kearon C, Akl EA, Ornelas J, et al. Antithrombotic therapy for VTE disease: antithrombotic therapy for VTE disease: CHEST guideline and expert panel report. Chest 2016; 149: 315-352.

3 Konstantinides SV, Torbicki A, Agnelli G, et al. 2014 ESC guidelines on the diagnosis and management of acute pulmonary embolism. Eur Heart J 2014; 35: 3033-3069.

4 Baglin $\mathrm{T}$, Luddington $\mathrm{R}$, Brown $\mathrm{K}$, et al. Incidence of recurrent venous thromboembolism in relation to clinical and thrombophilic risk factors: prospective cohort study. Lancet 2003; 362: 523-526.

5 Kearon C, Gent M, Hirsh J, et al. A comparison of three months of anticoagulation with extended anticoagulation for a first episode of idiopathic venous thromboembolism. N Engl J Med 1999; 340: 901-907.

6 Couturaud F, Sanchez O, Pernod G, et al. Two years versus six months of oral anticoagulation after a first episode of unprovoked pulmonary embolism. The PADIS-PE multicenter, double-blind, randomized, trial. JAMA 2015; 314: 31-40.

7 Agnelli G, Prandomi P, Becattini C, et al. Extended oral anticoagulant therapy after a first episode of pulmonary embolism. Ann Intern Med 2003; 139: 19-25.

8 Agnelli G, Prandoni P, Santamaria MG, et al. Three months versus one year of oral anticoagulant therapy for idiopathic deep vein thrombosis. N Engl J Med 2001; 345: 165-169.

9 Palareti G, Cosmi B, Antonucci E, et al. Duration of anticoagulation after isolated pulmonary embolism. Eur Respir J 2016; 47: 1429-1435.

10 Palareti G, Cosmi B, Legnani C, et al. D-dimer to guide the duration of anticoagulation in patients with venous thromboembolism: a management study. Blood 2014; 124: 196-203.

11 Palareti G, Cosmi B, Legnani C, et al. D-dimer testing to determine the duration of anticoagulation therapy. N Engl J Med 2006; 355: 1780-1789.

12 Kearon C, Spencer FA, O'Keeffe D, et al. D-dimer testing to select patients with a first unprovoked venous thromboembolism who can stop anticoagulant therapy: a cohort study. Ann Intern Med 2015; 162: 27-34.

13 Rodger MA, Kahn SR, Wells PS, et al. Identifying unprovoked thromboembolism patients at low risk for recurrence who can discontinue anticoagulant therapy. CMAJ 2008; 179: 417-426.

14 Carrier M, Le Gal G, Wells PS, et al. Systematic review: case-fatality rates of recurrent venous thromboembolism and major bleeding events among patients treated for venous thromboembolism. Ann Intern Med 2010; 152: 578-589.

15 Schulman S, Kearon C, Kakkar AK, et al. Extended use of dabigatran, warfarin, or placebo in venous thromboembolism. N Engl J Med 2013; 368: 709-718. 